УДК 532.783; 539.22

PACS 42.70.Df; 77.84.Nh; 05.70.Fn

\title{
Ориентационные переходы
}

\section{в магнитокомпенсированных ферронематиках с гомеотропным сцеплением частиц с матрицей}

\author{
Д. А. Петров, П. К. Скоков \\ Пермский государственный национальный исследовательский университет \\ 614990, Пермь, ул. Букирева, 15 \\ email: petrovda@bk.ru, skokovpk@gmail.com
}

\begin{abstract}
В рамках континуальной теории изучены индуцированные внешним магнитным полем ориентационные переходы в ферронематике - суспензии субмикронных частиц ферромагнетика, приготовленной на основе нематического жидкого кристалла. Рассматривался компенсированный ферронематик, в котором имелись равные доли феррочастиц с противоположно направленными магнитными моментами. В отсутствие магнитного поля такая суспензия является не намагниченной и представляет собой жидкокристаллический аналог антиферромагнетика. Учитывались следующие вклады в объемную плотность свободной энергии: энергия ориентационно-упругих деформаций поля директора, энергия диамагнитной матрицы и магнитных моментов феррочастиц в магнитном поле и вклад энтропии смешения идеального раствора частиц в суспензии. Рассмотрены мягкое гомеотропное сцепление феррочастиц с молекулами жидкого кристалла и мягкое планарное сцепление директора с границами слоя. Показано, что в исследуемой геометрии включение магнитного поля приводит к перераспределению магнитной примеси в слое ферронематика без появления искажений ориентационной структуры. Начальное антиферромагнитное упорядочение магнитных частиц сменяется ферримагнитным, т.е. происходит увеличение объемной доли частиц, ориентированных в направлении магнитного поля. С ростом магнитного поля однородное ферримагнитное состояние становится неустойчивым и происходит переход в неоднородную фазу. Последующее увеличение напряженности магнитного поля приводит к исчезновению искажений ориентационной структуры, вызывая переход ферронематика в однородную планарную фазу, в которой директор и намагниченность направлены вдоль магнитного поля. Аналитически найдены выражения для пороговых полей переходов между ориентационными фазами ферронематика для различных значений материальных параметров суспензии.
\end{abstract}

Ключевые слова: ферронематик; магнитная суспензия; мягкое сцепление; ориентационные переходы; эффект сегрегации

\section{Orientational transitions in the magnetocompensated ferronematics with the homeotropic coupling of particles with matrix}

\author{
D. A. Petrov, P. K. Skokov
}

Perm State University, Bukireva St. 15, 614990, Perm email: petrovda@bk.ru, skokovpk@gmail.com 
In the framework of the continuum theory the orientational transitions induced by an external magnetic field in the ferronematic i.e. suspension of submicron particles of ferromagnetic material on the basis of a nematic liquid crystal were studied. The ferronematic was assumed to be compensated i.e., having equal fractions of ferroparticles with oppositely directed magnetic moments. In the absence of magnetic field that suspension is not magnetized and it is a liquid-crystalline analog of an antiferromagnetic. We took into account the following contributions of the free energy to the bulk density: the potential of elastic deformation of the director field, the interactions of diamagnetic matrix and magnetic moments of ferroparticles with the magnetic field, as well as contribution of the entropy of mixing of the ideal solution of suspension particles. Soft homeotropic coupling of ferroparticles with the molecules of the liquid crystal and soft planar coupling of the director with boundaries of the compensated ferronematic layer were considered. It was shown that in considered geometry the applying magnetic field leads to a redistribution of the magnetic impurity in the layer of ferronematic without appearance of orientational structure distortions. The initial antiferromagnetic ordering of the magnetic particles is replaced by a ferrimagnetic one with increasing of the fraction of particles oriented in the magnetic field direction. With increasing of magnetic field the uniform ferrimagnetic state becomes unstable and Freedericksz transition appears. The subsequent increasing of the magnetic field leads to transition to the uniform angular phase, in which the director and magnetization are oriented in the direction of the magnetic field and distortions of orientational structure have been disappeared. Expressions for the threshold transition fields between orientational phases of ferronematic as functions of material parameters were found analytically.

Keywords: ferronematic; magnetic suspension; soft coupling; orientational transitions; segregation effect

Received 16.01.2017; accepted 10.05.2017

doi: 10.17072/1994-3598-2017-1-31-40

\section{1. Введение}

Жидкий кристалл (ЖК) представляет собой вещество, которое объединяет в себе свойства обычной жидкости (текучесть) и твердого кристаллического тела (анизотропия электрических, магнитных и оптических свойств).

Известно, что ЖК имеют малую анизотропию диамагнитной восприимчивости и для их переориентации в ограниченном объеме нужны достаточно большие магнитные поля ( 10 кГс) [1, 2]. Внедрение в ЖК-матрицу наноразмерных частиц ферромагнетика позволяет повысить ориентационный отклик такого композитного материала на внешнее магнитное поле. Впервые идея создания суспензий феррочастиц на основе нематического жидкого кристалла (НЖК) предложена в [3]. Описанный в [3] композитный материал получил название ферронематик (ФН).

По способу приготовления можно выделить два вида ФН: намагниченный и компенсированный. Если охлаждение ФН происходит в постоянном магнитном поле, то после перехода из изотропной фазы в ЖК состояние магнитные моменты частиц будут упорядочены вдоль одного направления и ФН получается намагниченным (ЖК аналог ферромагнетика) [3-13]. Если охлаждение проводить в отсутствие магнитного поля, то после перехода в ЖК состояние образующаяся суспензия является компенсированной, т.е. в ней имеются равные доли феррочастиц с противоположно направленными магнитными моментами [14-22]. Такая суспензия макроскопически не намагничена в отсутствие магнитного поля и является ЖК аналогом антиферромагнетика.

Для обычных ФН переход из начального однородного состояния (переход Фредерикса $[1,2])$ хорошо изучен, и он может происходить беспороговым [7, 8, 12, 13] и пороговым [9] образом и зависит от начальных взаимных ориентаций директора, намагниченности и магнитного поля. В свою очередь ориентационный отклик компенсированных суспензий изучен только для одного случая планарного сцепления молекул ЖК с границами ячейки и поверхностью феррочастиц [20-22].

Целью настоящей работы является исследование магнито-ориентационного отклика компенсированного ФН с мягким гомеотропным сцеплением магнитных частиц с ЖК-матрицей и мягким планарным сцеплением директора с границами слоя.

\section{2. Уравнения равновесного состояния ферронематика}

Ориентационная и магнитная структуры ФН в рамках континуальной теории описывается двумя векторными полями $\boldsymbol{n}$ и $\boldsymbol{m}[1,2]$. Единичный вектор $\boldsymbol{n}$ (директор) определяет направление преимущественной ориентации палочкообразных молекул ЖК, а единичный вектор намагниченно- 
сти $\boldsymbol{m}$ описывает среднюю ориентацию магнитных моментов примесных феррочастиц в суспензии.

Рассмотрим плоский слой ФН толщиной $L$. Начало координат поместим в середину слоя, ось $X$ направим вдоль оси легкого ориентирования $\boldsymbol{n}_{0}=(1,0,0)$, а ось $Z$ - ортогонально границам слоя (см. рис. 1). Магнитное поле $\boldsymbol{H}=(0,0, H)$ направим вдоль оси $Z$. Будем считать сцепление директора на границах слоя мягким и планарным, так что в отсутствие магнитного поля директор во всем слое параллелен оси легкого ориентирования $\boldsymbol{n} \| \boldsymbol{n}_{0}$. Сцепление директора с феррочастицами будем полагать мягким и гомеотропным, так что в отсутствие поля директор $\boldsymbol{n}$ и единичный вектор намагниченности $\boldsymbol{m}$ ортогональны $(\boldsymbol{n} \perp \boldsymbol{m})$.

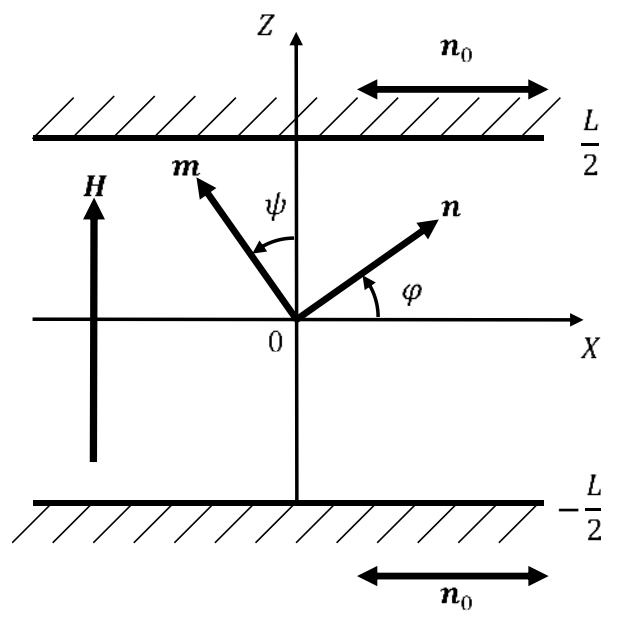

Рис. 1. Геометрия задачи

Полная свободная энергия слоя ФН в магнитном поле имеет вид $[3,4,20]$

$$
F=\int_{V} F_{V} d V+\int_{S} F_{S} d S,
$$

где объемная плотность свободной энергии

$$
\begin{gathered}
F_{V}=F_{1}+F_{2}+F_{3}+F_{4}+F_{5}, \\
F_{1}=\frac{1}{2}\left[K_{11}(\operatorname{div} \boldsymbol{n})^{2}+K_{22}(\boldsymbol{n} \cdot \operatorname{rot} \boldsymbol{n})^{2}+\right. \\
\left.+K_{33}(\boldsymbol{n} \times \operatorname{rot} \boldsymbol{n})^{2}\right], \\
F_{2}=-\frac{1}{2} \chi_{a}(\boldsymbol{n} \cdot \boldsymbol{H})^{2}, \quad F_{3}=-M_{s}\left(f_{+}-f_{-}\right) \boldsymbol{m} \cdot \boldsymbol{H}, \\
F_{4}=\frac{W_{p}}{d}\left(f_{+}+f_{-}\right)(\boldsymbol{n} \cdot \boldsymbol{m})^{2}, \\
F_{5}=\frac{k_{B} T}{v}\left(f_{+} \ln f_{+}+f_{-} \ln f_{-}\right) .
\end{gathered}
$$

Здесь $K_{11}, K_{22}$ и $K_{33}$ - константы Франка; $\chi_{a}>0$ - анизотропия диамагнитной восприимчивости ЖК; $M_{s}$ - намагниченность насыщения материала феррочастиц, $f_{+}$и $f_{-}$- объемные доли магнитных частиц с магнитными моментами $\boldsymbol{\mu}_{+}=M_{s} v \boldsymbol{m}$ и
$\boldsymbol{\mu}_{-}=-M_{s} v \boldsymbol{m}$, направленными в отсутствие магнитного поля параллельно и антипараллельно оси $Z$ соответственно; $W_{p}$ - поверхностная плотность энергии сцепления ЖК-матрицы с поверхностью магнитных частиц; $d$ - поперечный диаметр магнитной частицы; $v$ - объем магнитной частицы; $k_{B}$ - постоянная Больцмана; $T$ - температура. Мы предполагаем, что в отсутствие магнитного поля суспензия является компенсированной, т.е. $\left.f_{+}\right|_{H=0}=\left.f_{-}\right|_{H=0}=\bar{f} / 2$, где $\bar{f}=N v / V, N$ - число магнитных частиц в суспензии, $V$ - объем $Ф Н$. Концентрацию магнитной примеси будем считать малой $\bar{f} \ll 1$, что позволит не учитывать дипольдипольные взаимодействия.

Слагаемое $F_{1}$ представляет плотность свободной энергии ориентационно-упругих деформаций ЖК, $F_{2}$ учитывает энергию диамагнитной ЖКматрицы в магнитном поле и отвечает за квадрупольный механизм влияния магнитного поля на ориентационную структуру $\Phi Н, F_{3}$ - плотность свободной энергии взаимодействия феррочастиц с магнитным полем (дипольный механизм влияния магнитного поля на ориентационную структуру $\Phi Н), F_{4}$ - объемная плотность свободной энергии сцепления феррочастиц с ЖК-матрицей (для $W_{p}>0$ в отсутствие поля минимум этой энергии достигается при $\boldsymbol{n} \perp \boldsymbol{m}$, что соответствует гомеотропному сцеплению ЖК-матрицы с частицами), слагаемое $F_{5}$ учитывает вклад энтропии смешения идеального раствора частиц в суспензии.

Поверхностную плотность свободной энергии сцепления молекул ЖК с верхней и нижней границами слоя $F_{S}$ будем описывать с помощью потенциала Рапини [23]

$$
F_{S}=\frac{W}{2}\left(\left.\boldsymbol{n}\right|_{z= \pm L / 2} \times \boldsymbol{n}_{0}\right)^{2} .
$$

Для положительной величины плотности поверхностной энергии сцепления $W$ вклад (3) имеет минимум при $\boldsymbol{n} \| \boldsymbol{n}_{0}$, что отвечает планарному сцеплению директора на границах слоя.

Индуцированная магнитным полем деформация ориентационной структуры отвечает комбинациям поперечного и продольного изгиба и решение можно искать в виде

$$
\begin{gathered}
\boldsymbol{n}=(\cos \varphi(z), 0, \sin \varphi(z)), \\
\boldsymbol{m}=(-\sin \psi(z), 0, \cos \psi(z)) .
\end{gathered}
$$

После подстановки (4) в (1) получим

$$
\begin{gathered}
F=\int_{V}\left[\frac{1}{2}\left(K_{11} \cos ^{2} \varphi(z)+K_{33} \sin ^{2} \varphi(z)\right)\left(\frac{d \varphi}{d z}\right)^{2}-\right. \\
-\frac{1}{2} \chi_{a} H^{2} \sin ^{2} \varphi(z)-\mu_{s}\left(f_{+}-f_{-}\right) H \cos \psi(z)+ \\
+\frac{W_{p}}{d}\left(f_{+}+f_{-}\right) \sin ^{2}(\varphi(z)-\psi(z))+
\end{gathered}
$$




$$
\begin{gathered}
\left.+\frac{k_{B} T}{v} f_{+} \ln f_{+}+\frac{k_{B} T}{v} f_{-} \ln f_{-}\right] d V- \\
-\int_{S} \frac{W}{2} \cos ^{2} \varphi d S
\end{gathered}
$$

Используем следующие безразмерные величины

$$
\begin{gathered}
\tilde{z}=\frac{z}{L}, \quad \sigma=\frac{W_{p} \bar{f} L^{2}}{K_{11} d}, \quad \kappa=\frac{k_{B} T \bar{f} L^{2}}{K_{11} v}, \\
\omega=\frac{L W}{K_{11}}, \quad H_{q}=\frac{1}{L} \sqrt{\frac{K_{11}}{\chi_{a}}}, \quad H_{d}=\frac{K_{11}}{L^{2} M_{s} \bar{f}} \\
h=\frac{H}{H_{q}}, \quad b=\frac{H_{q}}{H_{d}}, \quad g_{ \pm}=\frac{f_{ \pm}}{\bar{f}}, \quad k=\frac{K_{33}}{K_{11}}
\end{gathered}
$$

и введем обозначение

$$
K(\varphi)=\cos ^{2} \varphi+k \sin ^{2} \varphi .
$$

Здесь $\tilde{z}$ - безразмерная координата (далее знак тильда будем опускать), $g_{+}$и $g_{-}-$приведенные объемные доли магнитных частиц с магнитными моментами, направленными параллельно и антипараллельно оси $Z$ соответственно. Параметр сегрегации $\kappa$ феррочастиц представляет собой меру интенсивности концентрационного расслоения магнитной примеси [3]. При $\kappa \gg 1$ распределение феррочастиц в слое ФН можно считать однородным, а при $\kappa \lesssim 1$ концентрационное расслоение дисперсной фазы становится существенным. Здесь $h$ - безразмерная напряженность магнитного поля, где в качестве единицы измерения поля выбрана величина $H_{q}$, определяемая из баланса плотности свободной энергии ориентационно-упругих деформаций $F_{1}$ и диамагнитного вклада $F_{2}$. Аналогичным образом можно ввести характерную величину поля $H_{d}$, которая определяется балансом вкладов $F_{1}$ и $F_{3}$. Параметр $b$ определяет относительный вклад квадрупольного и дипольного механизмов влияния магнитного поля на ориентационную структуру ФН и представляет собой отношение двух характерных магнитных полей $b=H_{q} / H_{d}$. Таким образом при $b>1$ появление деформации ориентационной структуры обусловлено преимущественно дипольным механизмом влияния магнитного поля, а при $b<1$ - квадрупольным. Наконец, $\omega$ - энергия сцепления молекул ЖК с верхней и нижней границами слоя и $\sigma-$ энергия сцепления магнитных частиц с ЖКматрицей.

Воспользуемся оценками безразмерных параметров, приведенных в работах [20, 21, 24]: $\kappa \approx 0.1 \div 1, \quad \sigma \approx 10^{-2} \div 1, \quad b \approx 10, \quad \omega \approx 1 \div 10$, $k \approx 1$.

После обезразмеривания свободная энергия ФН (5) примет вид

$$
\begin{gathered}
\tilde{F}=\frac{F L^{2}}{K_{11}}=\int_{-1 / 2}^{1 / 2}\left[\frac{1}{2} K(\varphi) \dot{\varphi}^{2}-\frac{1}{2} h^{2} \sin ^{2} \varphi-\right. \\
-h b\left(g_{+}-g_{-}\right) \cos \psi+\sigma\left(g_{+}+g_{-}\right) \sin ^{2}(\varphi-\psi)+ \\
\left.+\kappa\left(g_{+} \ln g_{+}+g_{-} \ln g_{-}\right)\right] d z- \\
-\left.\frac{\omega}{2} \cos ^{2} \varphi\right|_{z=1 / 2}-\left.\frac{\omega}{2} \cos ^{2} \varphi\right|_{z=-1 / 2}
\end{gathered}
$$

Здесь и далее дифференцирование по пространственной координате $Z$ будем обозначать точкой. Свободная энергия (8) представляет собой функционал относительно $\varphi(z), \psi(z), g_{+}(z)$ и $g_{-}(\mathrm{z})$. Минимизация (8) по переменным $\varphi(z)$ и $\psi(z)$ дает уравнения для угла ориентации директора и намагниченности

$$
\begin{gathered}
K(\varphi) \ddot{\varphi}+\frac{1}{2} \frac{d K(\varphi)}{d \varphi} \dot{\varphi}^{2}+\frac{h^{2}}{2} \sin 2 \varphi- \\
-\sigma\left(g_{+}+g_{-}\right) \sin (2 \varphi-2 \psi)=0, \\
\left(g_{+}-g_{-}\right) b h \sin \psi- \\
-\sigma\left(g_{+}+g_{-}\right) \sin (2 \varphi-2 \psi)=0 .
\end{gathered}
$$

Минимизация (8) по $g_{ \pm}$должна проводиться с условием постоянного числа частиц в системе

$$
\int\left(f_{+}+f_{-}\right) d V=N v
$$

или в безразмерной форме

$$
\int\left(g_{+}+g_{-}\right) d z=1 .
$$

В результате получим

$$
g_{ \pm}=Q \cdot \exp \left\{ \pm \frac{b h \cos \psi}{\kappa}-\frac{\sigma \sin ^{2}(\varphi-\psi)}{\kappa}\right\}
$$

где введено обозначение

$Q^{-1}=\int_{-1 / 2}^{1 / 2} 2 \exp \left(-\frac{\sigma \sin ^{2}(\varphi-\psi)}{\kappa}\right) \operatorname{ch}\left(\frac{b h \cos \psi}{\kappa}\right) d z$

После подстановки (11) в (10) получаем

th $\left(\frac{b h \cos \psi}{\kappa}\right) b h \sin \psi-\sigma \sin (2 \varphi-2 \psi)=0$.

Минимизация полной свободной энергии ФН по углу $\varphi$ приводит к следующим граничным условиям:

$$
\left.\left( \pm \frac{\partial \tilde{F}_{V}}{\partial \dot{\varphi}}+\frac{\partial \tilde{F}_{S}}{\partial \varphi}\right)\right|_{z= \pm 1 / 2}=0
$$

где $\tilde{F}_{V}$ и $\tilde{F}_{S}-$ безразмерные объемная и поверхностная плотности свободной энергии соответственно. Знак «+» отвечает верхней границе слоя, а знак «-»- нижней. Используя (8), условие (13) можно записать в виде 


$$
\left.\left(K(\varphi) \dot{\varphi} \pm \frac{\omega}{2} \sin 2 \varphi\right)\right|_{z= \pm 1 / 2}=0 .
$$

Проинтегрируем систему уравнений (9), (11) и (12) один раз. Для этого сначала умножим (9) на $\dot{\varphi}$, a $(10)$ на $\dot{\psi}$ и сложим, в результате получим

$$
\begin{gathered}
\dot{\varphi}=G(\varphi, \psi)^{1 / 2} K(\varphi)^{-1 / 2}, \quad z \in\left[-\frac{1}{2}, 0\right], \\
\dot{\varphi}=-G(\varphi, \psi)^{1 / 2} K(\varphi)^{-1 / 2}, \quad z \in\left[0, \frac{1}{2}\right] .
\end{gathered}
$$

Здесь введено обозначение

$$
\begin{aligned}
G(\varphi, \psi) & =h^{2}\left(\cos ^{2} \varphi-\cos ^{2} \varphi^{*}\right)- \\
& -2 \kappa\left(g_{+}+g_{-}-g_{+}^{*}-g_{-}^{*}\right),
\end{aligned}
$$

где $\varphi(0)=\varphi^{*}, \psi(0)=\psi^{*}$ и $g_{ \pm}\left(\varphi^{*}, \psi^{*}\right)=g_{ \pm}^{*}$ углы ориентации директора и намагниченности, а также функции распределения частиц в середине слоя соответственно.

Используя (15) и (16), граничные условия (14) можно переписать в виде

$$
\left.\left(G(\varphi, \psi)^{1 / 2} K(\varphi)^{1 / 2}-\frac{\omega}{2} \sin 2 \varphi\right)\right|_{z= \pm 1 / 2}=0 .
$$

Система уравнений (9), (11), (12) с граничными условиями (18) описывает равновесные состояния ФН во внешнем магнитном поле. Рассмотрим возможные однородные решения. В отсутствие магнитного поля $h=0$ в ФН нет искажений ориентационной и магнитной структуры $\varphi(z) \equiv \psi(z) \equiv 0$, т.е. директор направлен вдоль оси легкого ориентирования во всем слое, а единичный вектор намагниченности направлен параллельно оси $Z$ $(\boldsymbol{n} \perp \boldsymbol{m})$, что отвечает гомеотропному типу сцепления частиц и ЖК-матрицы. В этом состоянии приведенные объемные доли частиц с магнитными моментами, ориентированными параллельно и антипараллельно оси $Z$, равны $g_{+}=g_{-}=1 / 2$, а сам $Ф Н$ находится в компенсированной фазе, представляющей собой ЖК аналог антиферромагнетика.

Однородное состояние с $\varphi(z) \equiv \psi(z) \equiv 0$ остается устойчивым, пока магнитное поле не превысит некоторое пороговое значение $h_{c}$, выше которого появляются искажения ориентационной структуры. В полях $0<h<h_{c}$ ФН перестает быть компенсированным и происходит перераспределение феррочастиц между магнитными подсистемами согласно (11)

$$
g_{ \pm}=\frac{1}{2} \frac{\exp \{ \pm b h / \kappa\}}{\operatorname{ch}(b h / \kappa)}
$$

Из (19) видно, что величина $g_{+}$растет, а $g_{-}$ уменьшается с ростом поля, т.е. с включением магнитного поля ФН представляет собой ЖК аналог ферримагнетика с нескомпенсированными магнитными подсистемами.
Однородное состояние, описываемое решением $\varphi(z) \equiv \pi / 2$ и $\psi(z) \equiv 0$, становится устойчивым в магнитных полях, превышающих некоторое пороговое значение $h_{s}$. В этом состоянии директор и единичный вектор намагниченности ориентированы в направлении магнитного поля, т.е. $\boldsymbol{n}\|\boldsymbol{m}\| \boldsymbol{H}$, что отвечает планарному сцеплению феррочастиц с ЖК-матрицей - планарная фаза [25]. Нужно отметить, что распределение феррочастиц в планарной фазе также подчиняется выражению (19).

\section{3. Поля ориентационных переходов в ферронематике}

Как было сказано выше, однородное решение $\varphi(z) \equiv \psi(z) \equiv 0$ перестает быть устойчивым и появляются искажения ориентационной структуры, когда магнитное поле превышает значение $h_{c}$. По аналогии с чистым ЖК данный переход назовем переходом Фредерикса [1-2].

Вблизи перехода Фредерикса систему уравнений (9), (11), (12) можно линеаризовать по малым отклонениям директора от оси легкого ориентирования и намагниченности от направления поля, тогда в первом порядке разложения по $\varphi(z) \ll 1$ и $\psi(z) \ll 1$ получим

$$
\begin{gathered}
\ddot{\varphi}+h_{c}^{2} \varphi-2 \sigma \varphi+2 \sigma \psi=0, \\
\psi=\frac{2 \sigma}{2 \sigma+b h_{c} \operatorname{th}\left(b h_{c} / \kappa\right)} \varphi .
\end{gathered}
$$

Исключая из уравнения (20) угол $\psi(21)$, получаем

$$
\ddot{\varphi}+\lambda^{2} \varphi=0
$$

где

$$
\lambda^{2}=h_{c}^{2}-\frac{2 \sigma b h_{c} \operatorname{th}\left(b h_{c} / \kappa\right)}{2 \sigma+b h_{c} \operatorname{th}\left(b h_{c} / \kappa\right)} .
$$

Уравнение (22) нужно дополнить линеаризованными граничными условиями (14)

$$
\left.(\dot{\varphi} \pm \omega \varphi)\right|_{z= \pm 1 / 2}=0 .
$$

Решение уравнения (22) будем искать в виде

$$
\varphi=A \cos \lambda z+B \sin \lambda z,
$$

где $A$ и $B$ - некоторые константы. Из условия существования нетривиального решения (25) с помощью уравнений (24) найдем выражение для поля перехода Фредерикса

$$
\omega=\lambda \operatorname{tg} \frac{\lambda}{2} .
$$

На рис. 2 представлено решение уравнения (26) для различных значений параметров $\sigma, \kappa, \omega$ и $b$. Кривые на этом рисунке определяют границу перехода Фредерикса в ФН. Область под кривой отвечает однородному состоянию ФН с ферримагнитным упорядочением дисперсной фазы, планарным сцеплением директора с границами слоя $\boldsymbol{n} \| \boldsymbol{n}_{0}$ и гомеотропным сцеплением частиц с ЖК- 
матрицей $\boldsymbol{n} \perp \boldsymbol{m}$. Выше кривой находится неоднородная фаза, в которой угол между директором $\boldsymbol{n}$ и единичным вектором намагниченности $\boldsymbol{m}$ принимает значения от 0 до $\pi / 2$. Из рис. 2 , а видно, что в ФН, как и в случае чистого ЖК [15], поле Фредерекса увеличивается с ростом сцепления директора с границами слоя (рост $\omega$ ). Даже в предельном случае отсутствия сцепления директора с границами слоя $(\omega=0)$ наличие примеси стабилизирует ЖК-матрицу и с увеличением $\sigma$ требуются все большие магнитные поля для перехода в неоднородную фазу. Из рис. 2, б видно, что при переходе от квадрупольного режима влияния магнитного поля на ориентационную структуру ФН к дипольному (рост параметра $b$ ) для перехода Фредерикса требуются бо́льшие магнитные поля. Из рис. 2, в видно, что при дипольном режиме влияния магнитного поля $(b=5)$ сегрегационные эффекты слабо влияют на значение поля перехода Фредерикса, но для сильной сегрегации $(\kappa=0.1)$ поля $h_{\mathrm{c}}$ больше, чем для слабой $(\kappa=10)$. Из рис. 2 видно, что для представленных материальных параметров с увеличением энергии сцепления феррочастиц с ЖК-матрицей $\sigma$ происходит рост поля перехода Фредерикса.

Рассмотрим ряд предельных случаев. В отсутствие сцепления директора с границами слоя $(\omega=0)$ выражение (26) принимает вид

$$
\sigma=\frac{1}{2} \frac{b h_{c}^{2} \operatorname{th}\left(b h_{c} / \kappa\right)}{b \operatorname{th}\left(b h_{c} / \kappa\right)-h_{c}} .
$$

На рис. 2, а этому решению соответствует кривая $\omega=0$. При $\omega \rightarrow \infty$ (абсолютно жесткое сцепление директора с границами слоя) выражение (26) можно записать как

$$
\sigma=\frac{1}{2} \frac{b h_{c}\left(h_{c}^{2}-\pi^{2}\right) \operatorname{th}\left(b h_{c} / \kappa\right)}{b h_{c} \operatorname{th}\left(b h_{c} / \kappa\right)+\pi^{2}-h_{c}^{2}}
$$

На рис. 2, а этому решению соответствует кривая $\omega=\infty$. В случае слабого сцепления директора и намагниченности $(\sigma \rightarrow 0)$ выражение (28) можно представить в виде разложения в степенной ряд по малым $\sigma$

$$
\sigma=\frac{2 h_{c}\left(h_{c} \tan \left(h_{c} / 2\right)-\omega\right)}{h_{c} \tan ^{2}\left(h_{c} / 2\right)+2 \tan \left(h_{c} / 2\right)+h_{c}} .
$$

Как видно из (28) поле перехода Фредерикса в этом случае зависит только от энергий сцепления директора с границами слоя и поверхностью феррочастиц. В случае абсолютно жесткого сцепления директора и намагниченности выражение (26) примет вид

$$
\begin{aligned}
\omega & =\sqrt{h_{c}^{2}-b h_{c} \operatorname{th}\left(b h_{c} / \kappa\right)} \times \\
& \times \operatorname{tg}\left(\frac{\sqrt{h_{c}^{2}-b h_{c} \operatorname{th}\left(b h_{c} / \kappa\right)}}{2}\right) .
\end{aligned}
$$
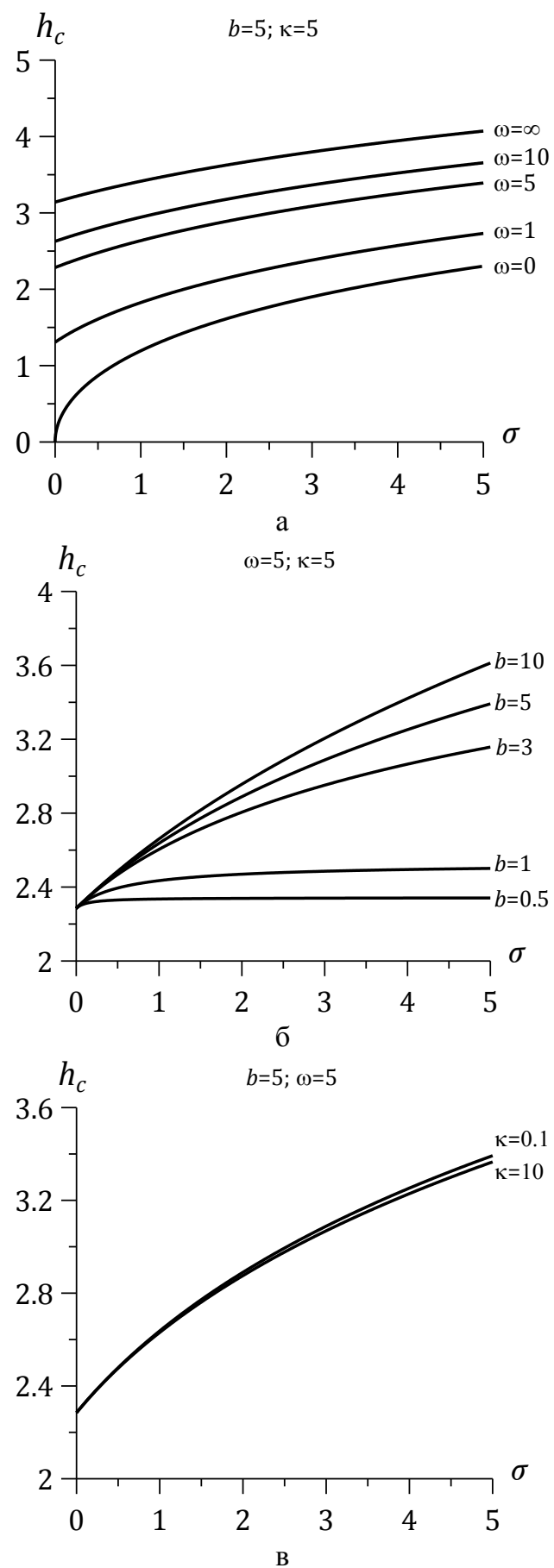

Рис. 2. Зависимости магнитного поля перехода Фредерикса $h_{c}$ от энергии сиепления феррочастии с ЖК-матрицей $\sigma$ для: $a-$ $b=5, \kappa=5$ и различных значений $\omega ; \sigma$ $\omega=5, \kappa=5$ и различных значений $b ; в-$ $b=5, \omega=5$ и различных значений $\kappa$

Как было сказано выше, система уравнений (9), $(11),(12)$ имеет однородное решение $\varphi(z) \equiv \pi / 2$ и $\psi(z) \equiv 0$ - планарная фаза, в которой директор и намагниченность ориентированы по полю $\boldsymbol{H}$. Это решение является устойчивым в магнитном поле, превышающем некоторое пороговое значение $h_{s}$. Вблизи перехода в планарную фазу отклонения директора и намагниченности от направления поля 
малы и угол $\varphi$ можно представить в следующем виде: $\varphi(z)=\pi / 2-\delta \varphi(z)$, где $\delta \varphi(z) \ll 1$. Учитывая, что $\psi(z) \ll 1$, разложение системы (9), (11), (12) в степенной ряд по малым $\delta \varphi$ и $\psi$ даст

$$
\begin{aligned}
& -k \delta \ddot{\varphi}+h_{s}^{2} \delta \varphi-\sigma(2 \delta \varphi+2 \psi)=0, \\
& \operatorname{th}\left(\frac{h_{s} b}{\kappa}\right) h_{s} b \psi-\sigma(2 \delta \varphi+2 \psi)=0 .
\end{aligned}
$$

Исключая $\psi$ из первого уравнения (30), получим

$$
\delta \ddot{\varphi}-\Lambda^{2} \delta \varphi=0,
$$

где введено обозначение

$$
\Lambda^{2}=\frac{1}{k}\left(h_{s}^{2}+\frac{2 \sigma b h_{s} \operatorname{th}\left(b h_{s} / \kappa\right)}{2 \sigma-b h_{s} \operatorname{th}\left(b h_{s} / \kappa\right)}\right) .
$$

Решение уравнения (31) будем искать в виде

$$
\delta \varphi=C \operatorname{ch}(\Lambda z)+D \operatorname{sh}(\Lambda z),
$$

где $C$ и $D$ - некоторые константы.

Линеаризуя граничные условия (14), получаем

$$
\left.(k \delta \dot{\varphi} \mp \omega \delta \varphi)\right|_{z= \pm 1 / 2}=0 .
$$

Подставляя выражение (33) в уравнения (34), находим выражение для поля перехода $Ф Н$ в планарную фазу $h_{s}$ :

$$
\omega=k \Lambda \operatorname{th} \frac{\Lambda}{2}
$$

На рис. 3 представлено решение уравнения (35), определяющие зависимость поля $h_{s}$ от энергии сцепления $\sigma$ для различных значений параметров $\omega, b$ и $\kappa$.

Кривые на рис. 3 определяют границу перехода ФН в планарную фазу. Области выше кривой отвечает планарная фаза, а ниже кривой - неоднородное состояние. На рис. 3 , а представлен случай разных значений энергии сцепления директора с границами слоя $\omega$; видно, что поле перехода в планарную фазу минимально для неограниченного образца $(\omega=0)$ и значительно увеличивается с ростом параметра $\omega$. В предельном случае абсолютно жесткого сцепления молекул ЖК с границами слоя $(\omega=\infty)$ переход ФН в планарную фазу возможен лишь при $h_{s}=\infty$. Из рис. 3,6 видно, что при смене режимов влияния магнитного поля от квадрупольного к дипольному, т.е. с ростом параметра $b$ для перехода в планарную фазу требуются меньшие магнитные поля. На рис. 3, в представлена зависимости поля $h_{s}$ от энергии сцепления $\sigma$ для промежуточного режима влияния магнитного поля $(b=1)$ и разных значений параметра сегрегации. В случае сильной сегрегации $(\kappa=0.1)$ нужны меньшие магнитные поля для перехода ФН в планарную фазу, чем для слабой $(\kappa=10)$ сегрегации магнитной примеси. Для дипольного режима влияния магнитного поля $(b=5)$ зависимости $h_{s}$ от $\sigma$ для $\kappa=0.1$ и $\kappa=10$ совпадают, т.е. поле перехода в планарную фазу не зависит от интен- сивности сегрегационных эффектов. Из рис. 3 также видно, что с увеличением энергии сцепления частиц с ЖК-матрицей поле перехода в планарную фазу увеличивается.

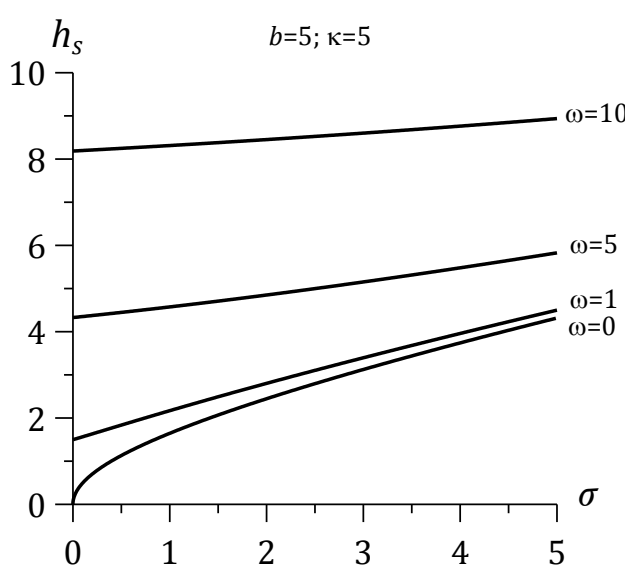

a)
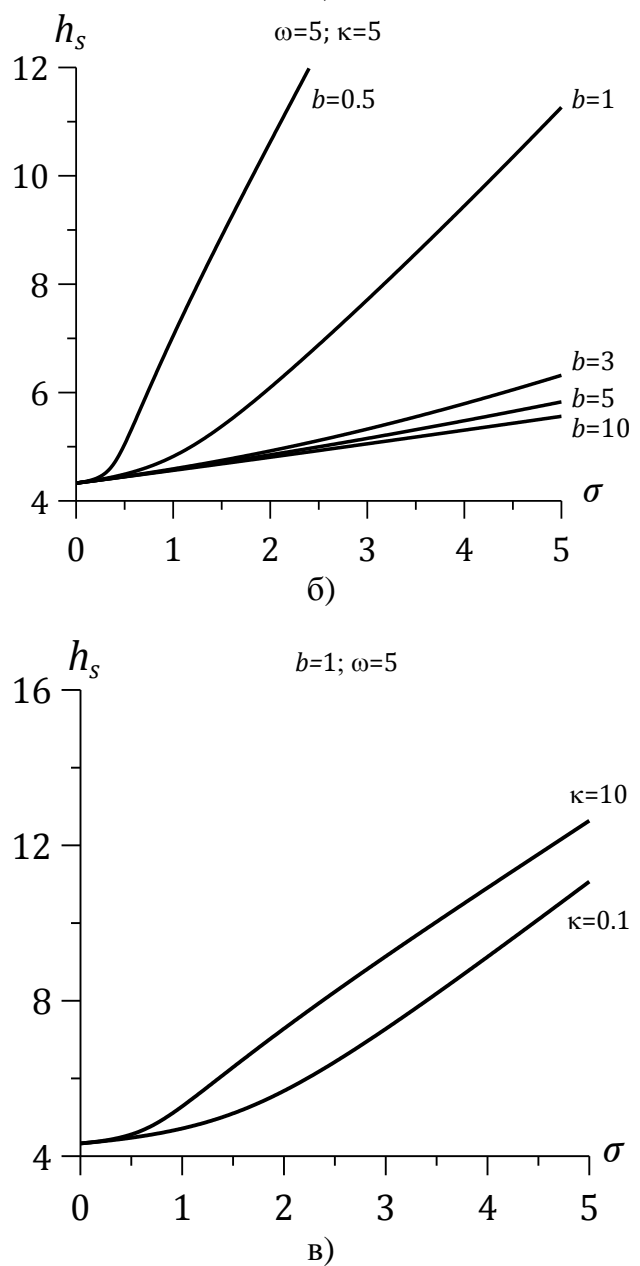

Рис. 3. Зависимости магнитного поля перехода в планарную фазу $h_{s}$ от энергии сиепления феррочастиц с ЖК-матрицей $\sigma$ для: $a-b=5, \kappa=5, k=1.5$ и различных значений $\omega ; \sigma-\omega=5, \kappa=5, k=1.5$ и различных значений $b$; в- $b=1, \omega=5, k=1.5 u$ различных значений $\kappa$ 
Рассмотрим ряд предельных случаев. В отсутствие сцепления директора с границами слоя $\omega=0$, выражение (35) примет вид

$$
\sigma=\frac{1}{2} \frac{b h_{s}^{2} \operatorname{th}\left(b h_{s} / \kappa\right)}{b \operatorname{th}\left(b h_{s} / \kappa\right)+h_{s}} .
$$

На рис. 3 , а этому решению соответствует кривая $\omega=0$.

Для слабого сцепления директора и намагниченности выражение (35) можно разложить в степенной ряд по малым $\sigma$ :

$$
\sigma=\frac{h_{s}\left(h_{s} \sqrt{k} \operatorname{sh}\left(h_{s} / \kappa\right)-\omega\left(1+\operatorname{ch}\left(h_{s} / \kappa\right)\right)\right)}{\sqrt{k} \operatorname{sh}\left(h_{s} / \kappa\right)+h_{s}} .
$$

В случае абсолютно жесткого сцепления директора и намагниченности $(\sigma \rightarrow \infty)$ выражение (35) примет вид

$$
\begin{aligned}
& \omega=\sqrt{k h_{s}\left(b \operatorname{th}\left(b h_{s} / \kappa\right)+h_{s}\right)} \times \\
& \times \operatorname{th}\left(\sqrt{\frac{k h_{s}\left(b \operatorname{th}\left(b h_{s} / \kappa\right)+h_{s}\right)}{4 k}}\right) .
\end{aligned}
$$

Следует отметить, что в случае слабого сцепления феррочастиц с ЖК-матрицей поле перехода в планарную фазу, как и поле перехода Фредерикса зависит только от энергий сцепления директора с частицами и границами слоя и не зависит от параметров $\kappa$ и $b$ (см. рис. 3 ).

\section{4. Заключение}

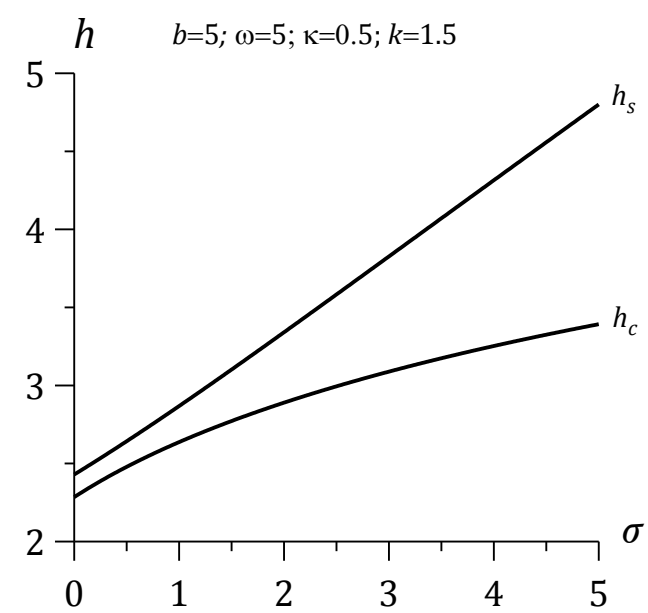

Рис. 4. Диаграмма ориентациионных фаз ФН

На рис. 4 представлена диаграмма ориентационных фаз ФН. Области под кривой $h_{c}$ отвечает однородная фаза ФН с гомеотропным сцеплением магнитных частиц с ЖК-матрицей $\boldsymbol{n} \| \boldsymbol{n}_{0}, \boldsymbol{n} \perp \boldsymbol{m}$, $\left(g_{+}>g_{-}\right)$. Между кривыми $h_{c}$ и $h_{s}$ находится область, соответствующая фазе с неоднородными распределениями директора и намагниченности. Области выше кривой $h_{s}$ отвечает однородная планарная фаза, в которой $\boldsymbol{n}\|\boldsymbol{m}\| \boldsymbol{H}$.
Подводя итог вышесказанного, нужно отметить, что магнито-ориентационный отклик компенсированных суспензий отличается от обычных намагниченных ФН. Как видно из выражения (19), чем выше интенсивность сегрегационных эффектов, тем быстрее происходит перераспределение частиц между магнитными подсистемами $g_{+}$и $g_{-}$ в сторону уменьшения последней.

\section{Список литературы}

1. Жен П.-Ж. де. Физика жидких кристаллов. М.: Мир, 1977. 400 с.

2. Stewart I. W. The Static and Dynamic Continuum Theory of Liquid Crystals: A Mathematical Introduction. London: Taylor \& Francis, 2004. 360 p.

3. Brochard F., de Gennes $P$. G. Theory of magnetic suspensions in liquid crystals // Journal de Physique. 1970. Vol. 31. P. 691-708.

4. Burylov S. V., Raikher Yu. L. Macroscopic properties of ferronematics caused by orientational interactions on the particle surfaces. II. Behavior of real ferronematics in external fields // Molecular Crystals and Liquid Crystals. 1995. Vol. 258. P. 123141.

5. Kopčanský P., Tomašovičová N., Koneracká M., Závišová V., Timko M., Džarová A., Šprincová A., Éber N., Fodor-Csorba K., Tóth-Katona T., Vajda A., Jadzyn J. Structural changes in the 6CHBT liquid crystal doped with spherical, rodlike, and chainlike magnetic particles // Physical Review E. 2008. Vol. 78. 011702.

6. Buluy O., Ouskova E., Reznikov Yu., Glushchenko A., West J., Reshetnyak $V$. Magnetically induced alignment of FNS // Journal of Magnetism and Magnetic Materials. 2002. Vol. 252. P. 159-161.

7. Zadorozhnii V. I., Reshetnyak V. Yu., Kleshchonok A. V., Sluckin T. J., Thomas K. S. Inverse Frederiks effect and bistability in ferronematic cells // Molecular Crystals and Liquid Crystals. 2007. Vol. 475. P. 221-231.

8. Zadorozhnii V. I., Sluckin T. J., Reshetnyak V. Yu., Thomas $K$. S. The Frederiks effect and related phenomena in ferronematic materials // SIAM Journal of Applied Mathematics. 2008. Vol. 68. P. 1688-1716.

9. Makarov D. V., Zakhlevnykh A. N. Tricritical phenomena at the Freedericksz transition in ferronematic liquid crystals // Physical Review E. 2010. Vol. 81.051710.

10. Podoliak N., Buchnev O., Buluy O., D'Alessandro G., Kaczmarek M., Reznikov Y., Sluckin T. J. Macroscopic optical effects in low concentration ferronematics // Soft Matter. 2011. Vol. 7. P. 47424749.

11. Mertelj A., Lisjak D., Drofenik M., Čopič M. Ferromagnetism in suspensions of magnetic platelets in liquid crystal // Nature. 2013. Vol. 504 P. 237241. 
12.Zakhlevnykh A. N., Petrov D. A. Weak coupling effects and re-entrant transitions in ferronematic liquid crystals // Journal of Molecular Liquids. 2014. Vol. 198. P. 223-233.

13.Zakhlevnykh A. N., Petrov D. A. Orientational bistability in ferronematic liquid crystals with negative diamagnetic anisotropy // Journal of Magnetism and Magnetic Materials. 2015. Vol. 393. P. 517-525.

14. Garbovskiy Yu. A., Glushchenko A. V. Liquid crystalline colloids of nanoparticles: preparation, properties, and applications // Solid State Physics. 2011. Vol. 62. P. 1-74.

15. Podoliak N, Buchnev O, Bavykin D.V., Kulak A.N., Kaczmarek M., Sluckin T.J. Magnetite nanorod thermotropic liquid crystal colloids: Synthesis, optics and theory // Journal of Colloid and Interface Science. 2012. Vol. 386. P. 158-166.

16. Tomašovičová N., Timko M., Mitróová Z., Koneracká M., Rajňak M., Éber N., Tóth-Katona T., Chaud X., Jadzyn J., Kopčanský P. Capacitance changes in ferronematic liquid crystals induced by low magnetic fields // Physical Review E. 2013. Vol. 87. 014501.

17. Gdovinová V., Tomašovičová N., Éber N., TóthKatona T., Závišová V., Timko M., Kopčanský P. Influence of the anisometry of magnetic particles on the isotropic-nematic phase transition // Liquid Crystals. 2014. Vol. 41. P. 1773-1777.

18. Petrov D. A., Zakhlevnykh A. N. Freedericksz transition in compensated ferronematic liquid crystals // Molecular Crystals and Liquid Crystals. 2012. Vol. 557. P. 60-72.

19. Захлевных А. Н., Петров Д. А. Влияние эффекта сегрегации на магнитные и оптические свойства компенсированного ферронематического жидкого кристалла // Журнал технической физики. 2012. Т. 82, вып. 9. С. 28-38.

20.Zakhlevnykh A. N., Petrov D. A. Magnetic field induced orientational transitions in soft compensated ferronematics // Phase Transitions. 2014. Vol. 87. P. 1-18.

21. Zakhlevnykh A. N., Petrov D. A. Orientational bistability and magneto-optical response in compensated ferronematic liquid crystals // Journal of Magnetism and Magnetic Materials. 2016. Vol. 401. P. 188-195.

22. Захлевных А. Н., Петров Д. А. Ориентационные переходы в антиферромагнитных жидких кристаллах // Физика твердого тела. 2016. Т. 58, вып. 9. С. 1841-1850.

23. Rapini A., Papoular M. Distorsion d'une lamelle nématique sous champ magnétique conditions d'ancrage aux parois // Journal de Physique Colloque. 1969. Vol. 30. P. 54-56

24. Blinov L. M., Chigrinov V. G. Electrooptic Effects in Liquid Crystal Materials. N. Y.: SpringerVerlag, 1994. 464 p.

25.Zakhlevnykh A. N. Threshold magnetic fields and Freedericksz transition in a ferronematic // Journal of Magnetism and Magnetic Materials. 2004. Vol. 269. P. 238-244.

\section{References}

1. de Gennes P. G. Physics of liquid crystals. Moscow: Mir, 1977. $400 \mathrm{c}$.

2. Stewart I. W. The Static and Dynamic Continuum Theory of Liquid Crystals: A Mathematical Introduction. London: Taylor \& Francis, 2004. 360 p.

3. Brochard F., Gennes de P. G. Theory of magnetic suspensions in liquid crystals. Journal de Physique, 1970, vol. 31, pp. 691-708.

4. Burylov S. V., Raikher Yu. L. Macroscopic properties of ferronematics caused by orientational interactions on the particle surfaces. II. Behavior of real ferronematics in external fields. Molecular Crystals and Liquid Crystals, 1995, vol. 258, pp. 123-141.

5. Kopčanský P., Tomašovičová N., Koneracká M., Závišová V., Timko M., Džarová A., Šprincová A., Éber N., Fodor-Csorba K., TóthKatona T., Vajda A., Jadzyn J. Structural changes in the 6CHBT liquid crystal doped with spherical, rodlike, and chainlike magnetic particles. Physical Review E, 2008, vol. 78, 011702.

6. Buluy O., Ouskova E., Reznikov Yu., Glushchenko A., West J., Reshetnyak V. Magnetically induced alignment of FNS. Journal of Magnetism and Magnetic Materials, 2002, vol. 252, pp. 159161 .

7. Zadorozhnii V. I., Reshetnyak V. Yu., Kleshchonok A. V., Sluckin T. J., Thomas K. S. Inverse Frederiks effect and bistability in ferronematic cells. Molecular Crystals and Liquid Crystals, 2007, vol. 475, pp. 221-231.

8. Zadorozhnii V. I., Sluckin T. J., Reshetnyak V. Yu., Thomas K. S. The Frederiks effect and related phenomena in ferronematic materials. SIAM Journal of Applied Mathematics, 2008, vol. 68, pp, 1688-1716.

9. Makarov D. V., Zakhlevnykh A. N. Tricritical phenomena at the Freedericksz transition in ferronematic liquid crystals. Physical Review E, 2010, vol. 81, 051710 .

10. Podoliak N., Buchnev O., Buluy O., D'Alessandro G., Kaczmarek M., Reznikov Y., Sluckin T. J. Macroscopic optical effects in low concentration ferronematics. Soft Matter, 2011, vol. 7, pp. 4742-4749.

11. Mertelj A., Lisjak D., Drofenik M., Čopič M. Ferromagnetism in suspensions of magnetic platelets in liquid crystal. Nature, 2013, vol. 504, pp. 237241.

12. Zakhlevnykh A. N., Petrov D. A. Weak coupling effects and re-entrant transitions in ferronematic liquid crystals. Journal of Molecular Liquids, 2014, vol. 198, pp. 223-233.

13.Zakhlevnykh A. N., Petrov D.A. Orientational bistability in ferronematic liquid crystals with nega- 
tive diamagnetic anisotropy. Journal of Magnetism and Magnetic Materials, 2015, vol. 393, pp. 517525.

14. Garbovskiy Yu. A., Glushchenko A. V. Liquid crystalline colloids of nanoparticles: preparation, properties, and applications. Solid State Physics, 2011, vol. 62, pp. 1-74.

15. Podoliak N, Buchnev O, Bavykin D. V., Kulak A. N., Kaczmarek M., Sluckin T.J. Magnetite nanorod thermotropic liquid crystal colloids: Synthesis, optics and theory. Journal of Colloid and Interface Science. 2012, vol. 386, pp. 158-166.

16. Tomašovičová N., Timko M., Mitróová Z., Koneracká M., Rajňak M., Éber N., Tóth-Katona T., Chaud X., Jadzyn J., Kopčanský P. Capacitance changes in ferronematic liquid crystals induced by low magnetic fields. Physical Review E, 2013, vol. 87, 014501 .

17. Gdovinová V., Tomašovičová N., Éber N., TóthKatona T., Závišová V., Timko M., Kopčanský P. Influence of the anisometry of magnetic particles on the isotropic-nematic phase transition. Liquid Crystals, 2014, vol. 41, pp. 1773-1777.

18. Petrov D. A., Zakhlevnykh A. N. Freedericksz transition in compensated ferronematic liquid crystals. Molecular Crystals and Liquid Crystals. 2012, vol. 557, pp. 60-72.

19. Zakhlevnykh A. N., Petrov D. A. Influence of the segregation effect on the magnetic and optical properties of a compensated ferronematic liquid crystal. Technical Physics, 2012, vol. 57, pp. 1208-1218.

20.Zakhlevnykh A. N., Petrov D. A. Magnetic field induced orientational transitions in soft compensated ferronematics. Phase Transitions, 2014, vol. 87, pp. 1-18.

21. Zakhlevnykh A. N., Petrov D. A. Orientational bistability and magneto-optical response in compensated ferronematic liquid crystals. Journal of Magnetism and Magnetic Materials, 2016, vol.401, pp. 188-195.

22. Zakhlevnykh A. N., Petrov D. A. Orientational Transitions in Antiferromagnetic Liquid Crystals. Physics of the Solid State, 2016, vol. 58, pp. 19061915.

23. Rapini A., Papoular M. Distorsion d'une lamelle nématique sous champ magnétique conditions d'ancrage aux parois. Journal de Physique Colloque, 1969, vol. 30, pp. 54-56.

24. Blinov L. M., Chigrinov V. G. Electrooptic Effects in Liquid Crystal Materials. New-York: SpringerVerlag, 1994. 464 p.

25. Zakhlevnykh A. N. Threshold magnetic fields and Freedericksz transition in a ferronematic. Journal of Magnetism and Magnetic Materials. 2004, vol. 269, pp. 238-244.

\section{Просьба ссылаться на эту статью в русскоязычных источниках следующим образом:}

Петров Д. А., Скоков П. К. Ориентационные переходы в магнитокомпенсированных ферронематиках с гомеотропным сцеплением частиц с матрицей// Вестник Пермского университета. Физика. 2017. № 1 (35). C. 31-40. doi: 10.17072/1994-3598-2017-1-31-40

\section{Please cite this article in English as:}

Petrov D. A., Skokov P. K. Orientational transitions in the magnetocompensated ferronematics with the homeotropic coupling of particles with matrix // Bulletin of Perm University. Physics, 2017, no. 1 (35), pp. 31-40. doi: 10.17072/1994-3598-2017-1-31-40 